\title{
Coefficients of static and dynamic friction of ceramic floor tiles: proposal of new method of surface roughness determination
}

\author{
Aline Demarch ${ }^{1,2}$, Elcio Angioletto ${ }^{1}$, Angela W. Vieira ${ }^{2}$, Andreza Dal Molin ${ }^{1}$, Oscar Rubem K. Montedo ${ }^{1, *}$, and \\ Elidio Angioletto ${ }^{1}$ \\ ${ }^{1}$ Universidade do Extremo Sul Catarinense - UNESC, Av. Universitária, 1105 - Bairro Universitário, 88806-000 Criciúma, SC, \\ Brazil \\ ${ }^{2}$ Eliane Revestimentos Cerâmicos, R. Maximiliano Gaidzinski, 245 - Centro, 88845-000 Cocal do Sul, SC, Brazil
}

Received: 2 November 2018 / Accepted: 16 March 2019

\begin{abstract}
Among the most important properties of ceramic floor tiles, the static and dynamic coefficients of friction assume an important role. The literature and current standards regarding ceramic floor tiles suggest different methods for determining the values of the coefficients of static and dynamic friction; however, all methods present limitations of implementation, and it is common for them to produce different values and hence specifications for different applications. In the present study, tests were conducted on the products with different topcoats and roughness to determine the coefficient of friction using the standards NBR 13818, ANSI A137.1, DIN 51130, and UNE-ENV 12633 and our proposed methodology. These values were compared with the roughness measurements obtained by mechanical contact profilometry. Additionally, empirical human evaluation of the roughness was performed. The trials indicated very different results on different experimental equipment for the same type of surface. The roughness test yielded parameters that correlate well with the empirical evaluation. The study shows the need for searching the uniformity of standards because each methodology produces different (even uncorrelated) results.
\end{abstract}

Keywords: Coefficient of static and dynamic friction / slip resistance / roughness of ceramic floor tiles / human empirical evaluation of roughness

\section{Introduction}

The use of ceramic floor tiles on external surfaces requires several different properties including slip resistance, based on the coefficient of friction (COF), which indicates the force opposing movement between two contact surfaces. This characteristic is extremely important because it determines the safety of pedestrians during movement over the ceramic coating. External areas are susceptible to the action of moisture, particulate material, and oil (among others), which makes the surface even smoother. These smooth surfaces cause slipping, resulting in numerous accidents [1].

Mechanical wear, the presence of liquid and particulate material, and the lack of maintenance strongly affect the $\mathrm{COF}$, and thus, the slip resistance [2]. These factors cause considerable local variations in the surface properties. The literature and ceramic floor tiles standards suggest different methods to determine the coefficients of static and dynamic friction; however, such methods present

* Corresponding author: oscar.rkm@gmail.com limitations of implementation [3]. The COF is one of the requirements to properly specify a ceramic tile, indicating its application. However, the current standard methodologies, which directly or indirectly determine the value of the COF, present serious inconsistencies. Thus, the COF of the same ceramic plate measured from the different methods available can present values so different that can be specified for different places. This can increase the risk for users and the manufacturer. This work compares the results of the COF obtained by the different existing methods and proposes a new method of measurement; such proposal is the scientific contribution for ceramic tiles users.

Therefore, this study aims to compare different methods for determining the coefficients of friction by applying different methodologies based on different standards, including NBR 13818 [4] that uses an equipment called Tortus, ANSI A137.1 [5] also known BOT method, DIN 51130 [6] that uses a ramp, UNE-ENV 12633 [7] that uses a pendulum, and propose a new method, which uses a movement sensor called Go!Motion.

The Tortus and BOT methods are similar. They consist of a motorized device that moves on the test surface 
(ceramic plate). There is a rubber attached to this device and connected to a sensor, which comes into contact with the surface and measures the force required for the rubber to move over that surface. The COF is then calculated. However, the test variables, such as the composition of the rubber and the speed of movement, are different between the devices, which consequently refer to different results. The pendulum method uses the energy loss of the pendulum movement that the test rubber suffers when in contact with the test surface. The ramp method measures the maximum angle of inclination to which a pedestrian can walk safely on the test surface; however, there is uncertainty of measurement because the test depends solely on human perception.

All of the current methodologies are useful. However, ISO does not determine a specific methodology for determining the COF of ceramic tiles and precisely for this reason, each organization proposes its own methodology. Thus, each evaluated methodology uses different measurement variables and therefore there is no agreement on which one is the most satisfactory.

Moreover, the relationship between rugosity parameters of tested surfaces and the values of friction coefficient measured from the different cited methods will be evaluated. Thus, we expect to clarify to the civil construction industry and the ceramic industry the differences among the different methods for determining the coefficients of friction and the best one to be used in the using conditions.

Slipping of a pedestrian is defined as the loss of control when walking owing to the reduction in contact with the foot support, shoeless or not, on a floor, which could cause a fall [8]. The slip resistance is related to the shoe sole surfaces and the floor surface; however, because the first cannot be known beforehand the slip resistance should be based on only the flooring $[9,10]$.

Devices that measure the COF of the shoe/contaminant/floor interface are used to evaluate slip resistance in various environments; however, the test conditions (such as the loading rate, time, normal force, velocity, and angle of the shoe) can affect the COF measurements. The nature of the relationships between these factors is not well understood [11]. The condition of the floor, the shoe, the inclination of the medium, and contaminants significantly affect the COF of the medium $[12,13]$. The angle of the shoe, its velocity, etc. are highly variable between devices and standards, resulting in different COF measurements for the same shoe/contaminant/ floor conditions [11].

In the last several years, there has been growing discussion on the disparity of slip resistance results from different devices [14]. Therefore, there is the need for an instrument to measure the slip resistance of surfaces that correlates with real results. Instruments that measure the coefficients of friction are complex and difficult to design; many have been developed, but none has been internationally accepted [15]. Many important aspects related to the reliability of the results given by the equipment require evaluation [16]. Studies show that, in the case of friction tests performed with portable equipment, even the data collection method can influence the results [17].
The measured COF can be dynamic or static; however, almost all researchers in Europe and North America who have studied the relationship between the COF and slipping agree that the coefficient of dynamic friction is more relevant than the coefficient of static friction [18].

The test methods can be different, depending on the tested surface, e.g., a ramp represents static and dynamic friction during human walking. Measurements that rely on an operator are highly uncertain at low angles but provide the only method of measuring friction on high-relief surfaces. A pendulum was used to simulate the effects of breaking car wheels on the road; it is portable, but it requires significant operator experience. Flat surfaces can be easily overestimated, whereas high-relief surfaces are difficult to measure. The measurement of the coefficient of dynamic friction can also be performed in situ using equipment such as the Tortus, which is less dependent on human influence but also leads to overestimation of flat surfaces and underestimation of certain relief depths (among other limitations). Therefore, the results should be treated carefully [19].

The subjective evaluation of the slip resistance of a coating can be sufficient to discriminate the differences between slippery floor surfaces [20]. Subjective human evaluation can correlate closely with the obtained slip resistance of a surface [21].

Although slip-resistant floors have been designed, contaminants can reduce the COFs, making slips and falls more likely to occur [20]. Studies indicate that contaminants such as sand particles can have a larger influence on the reduction of the COF than moisture [22]. Therefore, the designer should determine the appropriate product for all conditions of the design, considering the type of use, traffic, expected dirtiness, expected maintenance, expected wear, and the manufacturer's recommendations [16]. The COF should be within an optimal range so as providing ideal safety, comfort, and performance [23]. Another important consideration is product wear; in some cases, the slip resistance of a surface can be reduced over time because of a natural polishing process [24].

In addition to measurement of the COF, another characteristic that directly influences the slip resistance of a surface is roughness. Extremely smooth textures (those of low roughness) reduce the surface COF owing to the smaller contact area between the surface of the ceramic coating and the shoe soles. Therefore, the best known method of increasing slip resistance is to increase the surface roughness [25]. Knowledge of the roughness and undulation of the surface could provide a useful indication of the slip resistance of a wet floor [9], a characteristic that might be correlated with the COF [26], although, the relationship between the roughness profile and the value of the $\mathrm{COF}$ is not yet clear [27].

\section{Experimental procedures}

Samples with different finishes were used, i.e., smooth surfaces with glossy, satin, and polished finishes, and rough surfaces with gritted finishes and the application of corundum. The tested surfaces were subjectively evaluated 
Table 1. Test procedures.

\begin{tabular}{lllll}
\hline Method & Equipment & Brand & Standard & Friction \\
\hline BOT & BOT 3000-E & Regan Scientific & ANSI A137.1 & Static and dynamic \\
Proposed & - & - & - & Static and dynamic \\
Tortus & Tortus 3 & Wessex & NBR 13818 & Dynamic \\
Pendulum & SK 182 & Sassuolo Lab & UNE-ENV12633 & Dynamic \\
Ramp & Inclined ramp & - & DIN 51130 & Dynamic \\
\hline
\end{tabular}

through touching by three people, establishing a roughness scale. A representative sample of each type of ceramic plaque was evaluated and each test was repeated 12 times. The highest and lowest values were excluded from the analysis.

Tests with each of the previously described surfaces were also performed, following different measurement methodologies, some of which are standardized, to validate results with the human evaluation. The methodologies and the equipment used are presented in Table 1.

Except the proposed method, the other methods are well described in literature [28]; therefore, the description of the proposed method will be emphasized.

\subsection{Proposed methodology for obtaining the coefficient of kinetic friction $\left(\mu_{c}\right)$}

The specimen, of mass $m$, composed of a wooden block, of approximately $4 \mathrm{~cm}$ in width, $8 \mathrm{~cm}$ in length, and $4 \mathrm{~cm}$ in height and mass of $37.76 \mathrm{~g}$, which do not influence the result, since the COF is a property independent of these factors, coupled to a rubber sole sliding on a ceramic surface on an inclined plane, is under the action of the gravitational force and the friction force of the surface, as shown in Figure 1.

One of the steps in obtaining the coefficient of kinetic friction (dynamic friction) was performed by fitting a quadratic curve to a set of data obtained using a Go!Motion movement sensor (S, in Fig. 1) from Vernier ${ }^{\circledR}$. This fitting is possible because the motion of the specimen is described by the function:

$$
y=y_{0}+v_{0} t+\frac{1}{2} a t^{2}
$$

where $y$ is the position in a time $t$ (in $\mathrm{m}$ ), $y_{0}$ is the initial position (in $\mathrm{m}$ ), $v_{0}$ is the initial velocity (in $\mathrm{m} \mathrm{s}^{-1}$ ), and $a$ is the acceleration (in $\mathrm{m} \mathrm{s}^{-2}$ ).

The Go!Motion sensor, used in this work, is used to collect position, velocity, and acceleration data of moving objects. The Go!Motion was connected directly to a computer for real-time motion data collection during the test. The sensor presents an operating range from 0.15 to $6 \mathrm{~m}, 1 \mathrm{~mm}$ resolution, automatic temperature compensation, and $2 \mathrm{~mm}$ accuracy. The Go!Motion sensor emits short ultrasound sound waves from a transducer. These waves fill a cone-shaped area at about $15^{\circ}$ to $20^{\circ}$ of the axis of the beam centerline. The Go!Motion sensor captures the

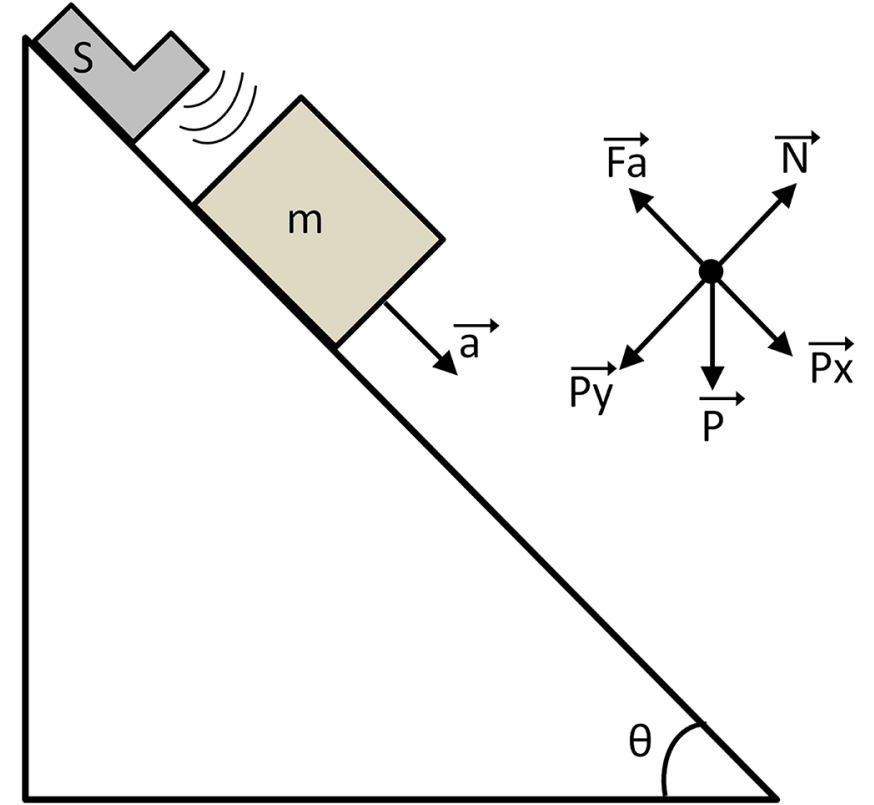

Fig. 1. Experimental apparatus for obtaining the coefficient of kinetic friction.

echo of these ultrasonic waves. The equipment measures how long it takes ultrasound waves to make the trip of the Go!Motion to the object. Using this time and the speed of sound in the air, the distance to the nearest object is determined. The error associated with the measurement performed by the sensor is in the third decimal place, so it is 0.001 .

For curve fitting, Logger Pro software was used, providing the value of the $\frac{1}{2}$ a parameter, which leads to the value of the acceleration of the block. The relationship between the acceleration and the COF is given by Newton's second law.

$$
P_{x}-F_{a}=m * a,
$$

where $P_{x}$ is the component weight in the $x$ axis $(\mathrm{N}), F_{a}$ is the force of dynamic friction $(\mathrm{N}), m$ is the mass of the body $(\mathrm{kg})$, and $a$ is the acceleration (in $\mathrm{ms}^{-2}$ ).

Substituting the terms, it is possible to isolate the coefficient of kinetic friction $\left(\mu_{c}\right)$ :

$$
\mu_{c}=\frac{P * \sin \theta-m * a}{P * \cos \theta}
$$




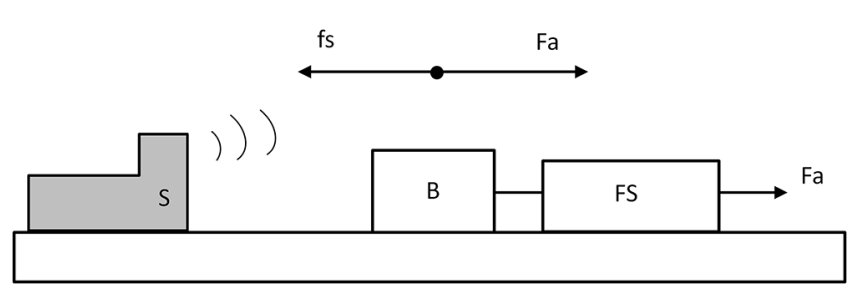

Fig. 2. Experimental apparatus for obtaining the coefficient of static friction.

where $P$ is the weight of the bloc $(\mathrm{N}), \theta$ is the angle of inclination of the inclined plan (degree), $m$ is the mass of the body $(\mathrm{kg})$, and $a$ is the acceleration (in $\mathrm{m} \mathrm{s}^{-2}$ ).

Thus, using the value of acceleration measured by the motion sensor, the mass of the object, and the inclination angle $\theta$ indicated in Figure 1, it is possible to determine the $\mathrm{COF}$ of the surface material on the inclined plane.

\subsection{Proposed methodology for the coefficient of static friction $\left(\mu_{s}\right)$}

The specimen (B) is pulled over a horizontal ceramic surface (Fig. 2) by a force $F_{a}$ applied by the experimenter using a dual-range force sensor (FS) from Vernier ${ }^{\circledR}$. The sensor, monitored using Logger Pro, records the force required to make the block slide. The motion sensor (S) indicates the exact moment when the force applied equals the maximum force of static friction $\left(f_{s}\right)$.

The force of friction is obtained by reading the graph of force versus time, calibrated based on the time at which the block starts to move, as indicated by the motion sensor.

The normal force $N$ is determined based on the mass of the block, measured on a digital scale of $0.01 \mathrm{~g}$ accuracy. In this way, the coefficient of static friction can be calculated from the following equation:

$$
\mu_{e}=\frac{F_{a}}{N}
$$

The test results were compared to roughness measurements obtained though mechanical contact profilometry, using a Mitutoyo profilometer, model SJ201-P. The measurements were repeated 12 times for each piece; we excluded the highest and the lowest values obtained.

The developed methods for both the determination of the coefficient of static friction and the determination of the coefficient of dynamic friction use analysis variables different from those analyzed by the current methodologies, such as the test rubber material and the results. In this way, it is expected to obtain also different results. It can be observed that in the methodology proposed for the dynamic test, the movement occurs due to the force of gravity acting on the mass, according to Figure 1.

\section{Results and discussion}

Based on human analysis, it was possible to define the roughness scale of the surfaces, as shown in Table 2, where
Table 2. Empirical evaluation of the tested surfaces.

\begin{tabular}{ll}
\hline Surface & Scale \\
\hline Polished smooth & 1 \\
Glossy smooth & 2 \\
Satin smooth & 3 \\
Corundum rough & 4 \\
Gritted rough & 5 \\
\hline
\end{tabular}

Table 3. Pearson coefficient for sensory analysis versus analyzed methods.

\begin{tabular}{ll}
\hline Method & $\begin{array}{l}\text { Pearson } \\
\text { coefficient }\end{array}$ \\
\hline Dry static - BOT & 0.717 \\
Wet static - BOT & 0.791 \\
Dry dynamic - BOT & 0.364 \\
Wet dynamic - BOT & 0.879 \\
Dry dynamic - Tortus & 0.765 \\
Wet dynamic - Tortus & 0.467 \\
Dry ramp (oil) & 0.538 \\
Wet ramp (oil) & 0.911 \\
Pendulum & 0.916 \\
Dry static - developed method & -0.789 \\
Wet static - developed method & 0.119 \\
Wet dynamic - developed method & 0.965 \\
\hline
\end{tabular}

the smoothest product is ranked 1 , and the roughest is ranked 5 (all participants agreed on this ranking). Observations indicated that the polished and glossy surfaces have very similar textures. Having established this scale, it is easier to compare the results from different equipment with the human evaluation. The results were divided into dynamic and static and dry and wet conditions. The test for the coefficient of static friction was performed using only the proposed method and the BOT equipment.

Table 3 shows the Pearson correlation coefficient between the sensory analysis and the results obtained with each of the analyzed methods. The methodology that has presented the highest correlation with the sensory analysis, performed with Pearson coefficient equal to 0.965, has been chosen to be developed in wet and dynamic test conditions.

In addition to Pearson's correlation, we performed a one-way analysis of variance, relating the empirical evaluation of texture to the results obtained by all methods analyzed. For all cases, the obtained $p$-value was lower than 0.001 , that is, by evaluating the data with a $95 \%$ confidence index, it can be stated that the texture of the surfaces has a significant influence on the friction results in all methodologies tested. 


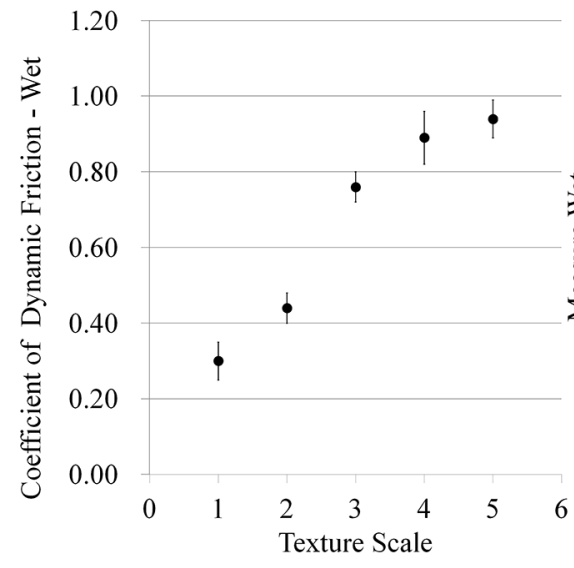

Proposed method

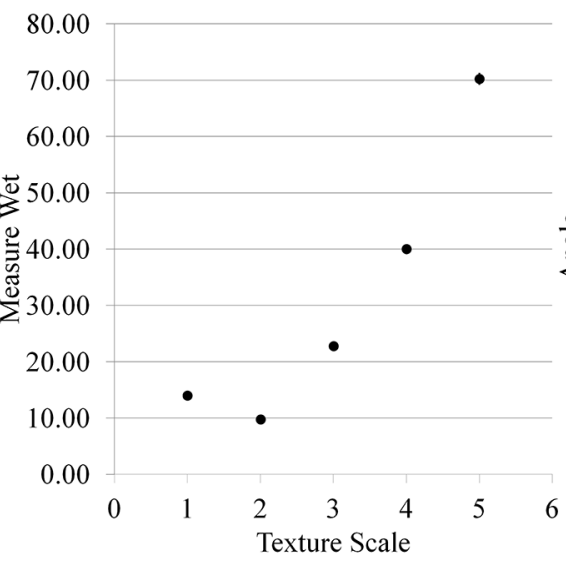

Pendulum

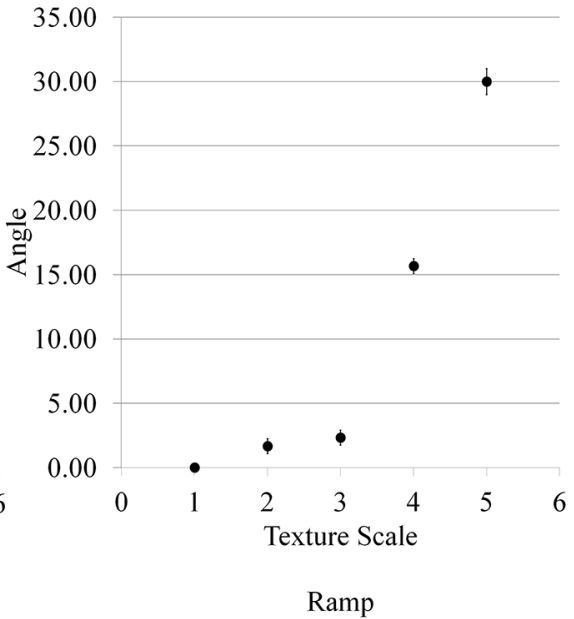

Ramp

Fig. 3. Relationship between texture scale and obtained results.

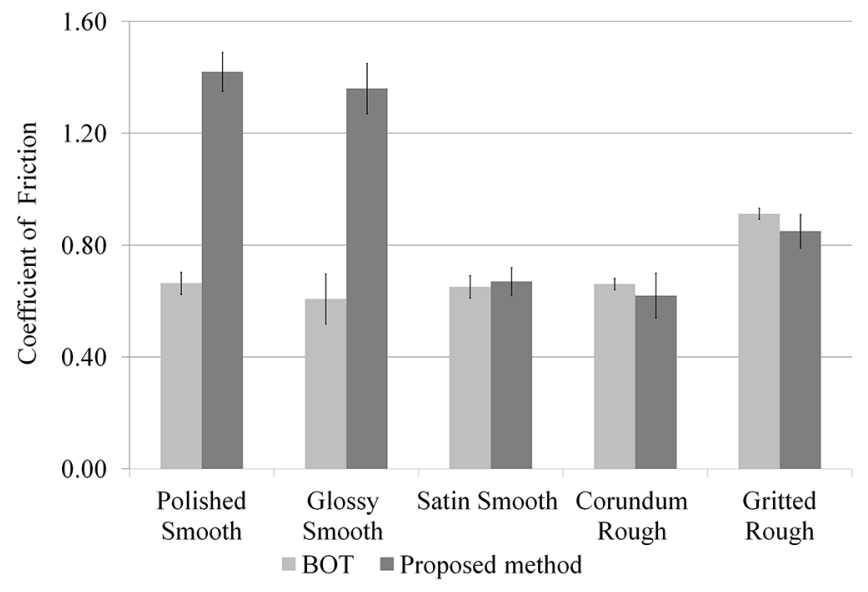

(a)

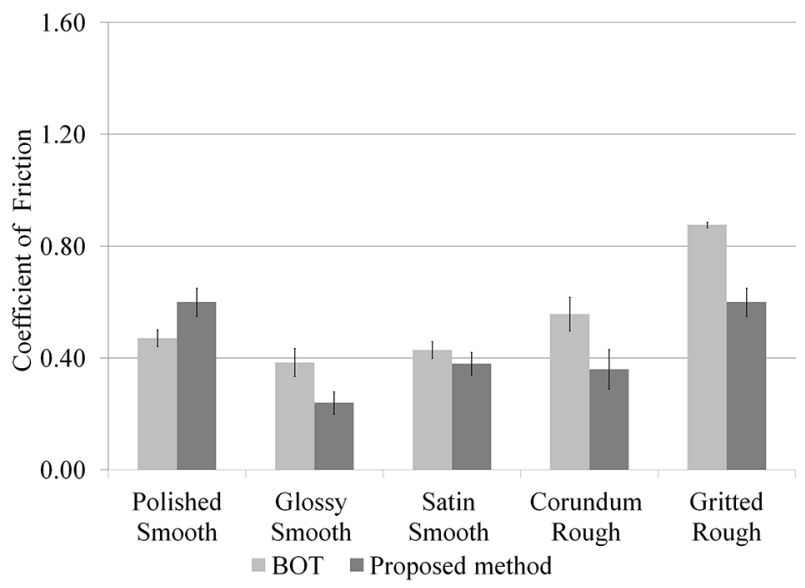

(b)

Fig. 4. Coefficient of static friction: (a) dry condition and (b) wet condition.

According to Table 3 and figures that will be presented next, a great variability in the results is observed. This situation is due to the fact that each of the studied methodologies uses different test variables, such as rubbers having different compositions, and consequently different hardness and elasticity, among other characteristics. In addition, the employed measuring principle is also not the same as previously described; each method uses a different physical principle to perform the measurements. These factors make each of the tests generate quite different results.

Figure 3 shows the performance of the methodologies that presented the highest Pearson coefficient. One can observe a great correlation between the variables.

As observed in Figure 4, for both the wet and dry conditions, it is impossible to establish a correlation between results obtained using different equipment. In the proposed method, there is a discrepancy among results wherein the two smoothest surfaces show higher values. On comparison with the sensory scale, it was observed that the results did not correlate for the two testing equipment setups. The error bars shown in the graph of Figure 3
Table 4. Pearson coefficient for correlation of static coefficient.

\begin{tabular}{lll}
\hline & $\begin{array}{l}\text { Dry static }- \\
\text { BOT }\end{array}$ & $\begin{array}{l}\text { Wet static - } \\
\text { BOT }\end{array}$ \\
\hline $\begin{array}{l}\text { Dry static - developed } \\
\text { method }\end{array}$ & -0.26 & -0.35 \\
$\begin{array}{l}\text { Wet static - developed } \\
\text { method }\end{array}$ & 0.68 & 0.634 \\
\hline
\end{tabular}

correspond to the standard deviation of 10 measurements performed with each ceramic plate in each equipment/ methodology.

Table 4 shows the Pearson coefficient, which relates the results obtained in the test normalized by ANSI and the developed methodology.

The results show that there is practically no correlation between the methods in the dry condition. In wet condition, the correlation is slightly higher, but still small. 


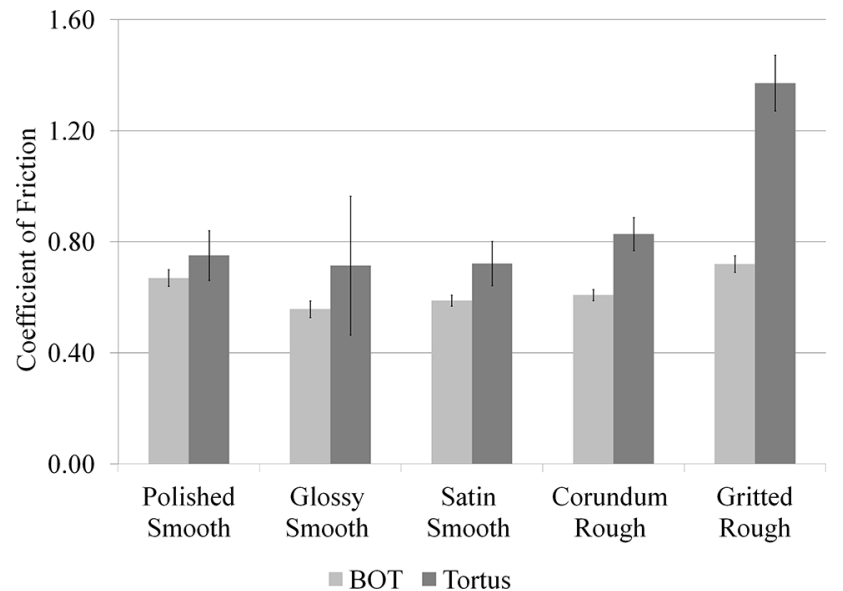

(a)

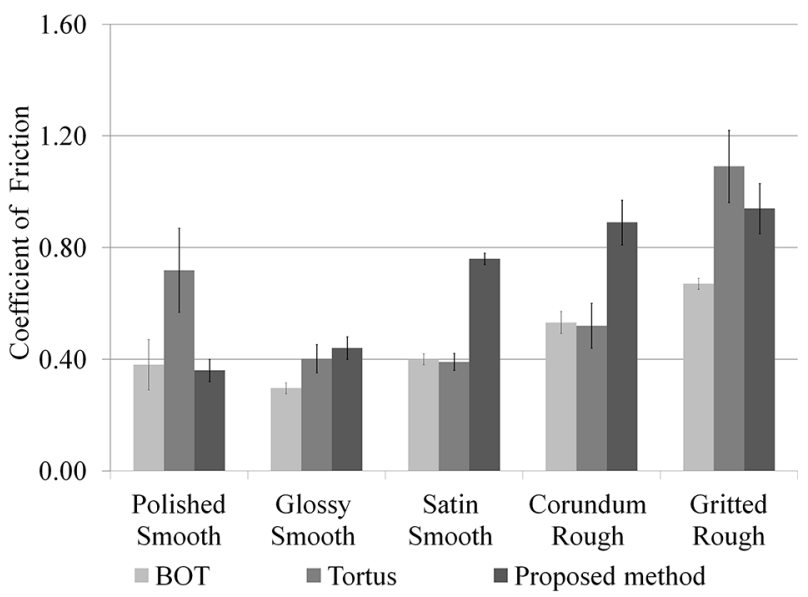

(b)

Fig. 5. Coefficient of dynamic friction: (a) dry condition and (b) wet condition.

Table 5. Pearson coefficient - correlation of dynamic coefficient.

\begin{tabular}{ll}
\hline Method & $\begin{array}{l}\text { Wet dynamic - } \\
\text { developed method }\end{array}$ \\
\hline Dry dynamic - BOT & 0.290 \\
Wet dynamic - BOT & 0.838 \\
Dry dynamic - Tortus & 0.627 \\
Wet dynamic - Tortus & 0.337 \\
Dry ramp & 0.542 \\
Wet ramp (oil) & 0.810 \\
Wet pendulum & 0.846 \\
\hline
\end{tabular}

Table 6. Results of the ramp and pendulum tests.

\begin{tabular}{llll}
\hline Product & $\begin{array}{l}\text { Wet } \\
\text { ramp } \\
\text { (oil) }\end{array}$ & Pendulum & Suggestion of using \\
\hline $\begin{array}{l}\text { Polished smooth } \\
0^{\circ}\end{array}$ & 14 & Internal places \\
$\begin{array}{l}\text { Glossy smooth } \\
\text { Satin smooth }\end{array}$ & $2^{\circ}$ & 10 & Internal places \\
$\begin{array}{l}\text { Corundum } \\
\text { rough }\end{array}$ & $16^{\circ}$ & 40 & Internal places \\
Gritted rough & $30^{\circ}$ & 70 & $\begin{array}{l}\text { External places } \\
\text { (without inclination) } \\
\text { External places } \\
\text { (with inclination) }\end{array}$ \\
\hline
\end{tabular}

Table 7. Roughness parameters.

\begin{tabular}{llllll}
\hline Parameter & Polished smooth & Glossy smooth & Satin smooth & Corundum rough & Gritted rough \\
\hline$R_{\mathrm{a}}(\mu \mathrm{m})$ & 0.44 & 1.86 & 6.31 & 22.60 & 21.01 \\
$R_{\mathrm{y}}(\mu \mathrm{m})$ & 13.66 & 10.03 & 33.35 & 122.36 & 140.37 \\
$R_{\mathrm{z}}(\mu \mathrm{m})$ & 6.45 & 6.78 & 23.29 & 81.52 & 95.98 \\
$R_{\mathrm{q}}(\mu \mathrm{m})$ & 1.03 & 2.28 & 7.69 & 27.87 & 26.33 \\
\hline
\end{tabular}

The results for the coefficient of dynamic friction are shown in Figure 5. The results obtained with BOT equipment using the ASTM standard and the proposed methodology in the wet condition show good correspondence with the human evaluation. The same cannot be said about Tortus, which showed very high values for the COF of the polished surface.

Table 5 shows the correlation established by the Pearson coefficient for the results of the standardized methodologies and the developed method. The coefficients show that there is a good correlation among the results obtained with the BOT, the ramp, and the pendulum, all of them obtained in the wet condition.
The results of the test with the pendulum and the ramp cannot be correlated with the values obtained using other equipment because these tests report angles and not absolute values of the COF. Table 6 presents the angles obtained from each equipment.

The results from the two equipment setups present a better correlation with the established sensory scale.

Regarding the roughness results, as represented in Table 7, the rough products show higher $R_{\mathrm{a}}$ values than the smooth ones; this parameter indicates the arithmetic average of the absolute relief height values, i.e., rougher products have more prominent reliefs. 
Parameters $R_{\mathrm{y}}$ and $R_{\mathrm{z}}$ are related to the amplitude of the roughness profile; they indicate the height of the troughs and peaks, i.e., re-entrances and saliences.

These parameters also show very high correlations with the established empirical scale, the highest the value of its parameters, the highest amplitude of re-entrances and saliences and, therefore, the highest roughness and ruggedness of the surface.

The polishing process is designed to eliminate any surface salience; thus, it presented lower values for most of the parameters when compared to the other surfaces. The grit and corundum are added to the surface of the ceramic coating to increase saliences and recesses; therefore, they have higher roughness parameters, as compared to the other surfaces.

\section{Conclusions}

Most of the methods show divergent results that, at times, disagree with the empirical human evaluation of roughness and smoothness. The evaluation of the coefficient of static friction did not result in a good correlation between methods; regarding dynamic friction, the test performed with the Tortus presented the most satisfactory correlation both with the other tests and with the sensory evaluation. The roughness test produced parameters that correlate with human sensory evaluation; however, because it is not an evaluation method based on a standard, it is rarely applied. The results of this study indicate the need to establish a single method and unify standards as each methodology can produce a different result. By critically evaluating the obtained results and comparing the different used methods, some considerations can be formulated to explain such discrepancies. All the evaluated methods, including the proposed one, use the contact of a piece of rubber, which is the sliding surface, with the surface of the ceramic sample in the evaluation. Each method uses a characteristic geometry and a dynamic motion. By comparing the methods tested in this work with the sensorial analysis, it was concluded that the method developed for the determination of the coefficient of dynamic friction in wet condition was that one that presented higher correlation with the Pearson coefficient (value equal to 0.965 ). It should also be noted that the rubbers show different textures, hardness, and chemical composition. Such differences are translated into the different obtained results. In the analysis of many researchers, the roughness method gives a greater guarantee to the ceramic floor in a specific place of use, since the methods that measure the COF take into account the contact between two surfaces. This can induce errors in the specification of use.

\section{Implications and influences}

The ceramic tiles market was valued at USD 215 Billion in 2017 and is projected to reach USD 320 Billion by 2023. Its importance in the world and the existing challenges, particularly the technical ones, require permanent research and development.

There are many methods to determine the characteristics of ceramic tiles in order to determine the quality and applicability of ceramic tiles. Some of them is related to security and reliability, such as the determination of surface roughness in order to allow the correct specification.

Such method brings some uncertainty and new alternatives play an important role in this industrial sector.

This work presents a proposal of new method of surface roughness determination for ceramic tiles. The roughness test yielded parameters that correlate well with the empirical evaluation. The comparative study shows that the roughness method gives a greater guarantee to the ceramic floor in a specific place of use.

Because of this, the authors believe that this work may bring a great interest to the readers of International Journal of Metrology and Quality Engineering.

\section{References}

1. S. Derler et al., Saf. Sci. 76, 12 (2015)

2. I.-J. Kim, H. Hsiao, P. Simeonov, Appl. Ergon. 44, 58 (2013)

3. J. Kim, Saf. Health Work 3, 22 (2012)

4. Associação Brasileira de Normas Técnicas, NBR 13818 Anexo A - Determinação do Coeficiente de Atrito: Placas Cerâmicas para Revestimento - Especificações e Métodos de Ensaios, Rio de Janeiro, 1997

5. American National Standards Institute, A137.1: American National Standard Specifications for Ceramic Tile, Clemson, 2012

6. Deutsches Institut Für Normung, DIN 51130: Testing of floor coverings - Determination of the anti-slip properties, Berlin, 2004

7. UNE-ENV 12633:2003: Method of determination of unpolished and polished slip/skid resistance value, 2003

8. C. Wetzel, Ph.D. thesis, Bergische Universität Wuppertal, 2012

9. G.W. Harris, S.R. Shaw, J. Occup. Accidents 9, 287 (1988)

10. I.-J. Kim, Wear 360-361, 77 (2016)

11. K.E. Beschorner et al., Appl. Ergon. 38, 773 (2006)

12. K.W. Li, W.-R. Chang, C.-C. Chang, Saf. Sci. 47, 1434 (2010)

13. L. Liu et al., Saf. Sci. 48, 1321 (2010)

14. The measurement of the slip resistance of floor surfaces - the Tortus and the Pendulum, Constr. Build. Mater. 2, 163 (1988)

15. L. Strandberg, Ergonomics 28, 131 (1985)

16. R. Bowman, Proceedings of Qualicer, Espanha, 2016

17. S. Matz, R. Gronqvist, Saf. Sci. 42, 483 (2004)

18. R. Skiba, A. Kuschefski, N. Cziuk, Entwicklung eines normgerechten Prfifverfahrens zur Ermittlung der Gleitsicherheit von Schuhsohlen, Schriftenreihe der Bundesanstalt ffir Arbeitsschutz, Dortmund, Fb Nr 526 (1987)

19. M. Engels, Proceedings of Qualicer, Espanha, 2016

20. R. Myung, J.L. Smith, T.B. Leamon, Int. J. Ind. Ergon. 11, 313 (1993) 
21. K.W. Li et al., Saf. Sci. 42, $547(2004)$

22. K.W. Li et al., Saf. Sci. 45, 980 (2007)

23. T. Zamora et al., Int. J. Ind. Ergon. 41, 410 (2011)

24. S. Derler, F. Kausch, R. Huber, Saf. Sci. 43, 751 (2005)
25. B.S. Parra et al., Cer. Ind. 11, 15 (2006)

26. W.-R. Chang et al., Appl. Ergon. 41, 27 (2010)

27. W.-R. Chang, Saf. Sci. 40, 577 (2002)

28. M.M. Scorisa et al., Cer. Ind. 21, 7 (2016)

Cite this article as: Aline Demarch, Elcio Angioletto, Angela W. Vieira, Andreza Dal Molin, Oscar Rubem K. Montedo, Elidio Angioletto, Coefficients of static and dynamic friction of ceramic floor tiles: proposal of new method of surface roughness determination, Int. J. Metrol. Qual. Eng. 10, 4 (2019) 